\title{
EVALUACIÓN DE LA PRUEBA DE LA MONEDA PERUANA EN EL TAMIZAJE DE TRASTORNO COGNITIVO EN ADULTOS MAYORES
}

\author{
Teodoro J. Oscanoa ${ }^{1, a}$, Edwin Cieza², José F. Parodi1,a, Napoleón Paredes ${ }^{2, a}$
}

\begin{abstract}
RESUMEN
Objetivos. Adaptación peruana del test de la moneda en el tamizaje de trastorno cognitivo en adultos mayores $(\geq 60$ años). Materiales y métodos. El presente es un estudio de fase I de evaluación de prueba diagnóstica con muestreo de conveniencia y calculo de sensibilidad y especificidad, con una prevalencia pretest de $50 \%$. Para la definición operacional de pacientes con trastorno cognitivo se utilizó los criterios de la DSM IV (The Diagnostic and Statistical Manual of Mental Disorders) y el Global Deterioration Scale (GDS) (controles: GDS 1 y 2; casos: mayor de 2). Para encontrar el punto de corte se utilizó la curva ROC (Receiver Operating Characteristic). Resultados. Los pacientes estudiados fueron 42 casos y 42 controles, no hubo diferencia significativa entre la edad $(77,88 \pm 6,01$ y $76,14 \pm 6,49$ años) y años de educación $(13,69 \pm 3,70$ y $8,17 \pm 4,71)$. La versión peruana del "eurotest" denominada "prueba de la moneda peruana" tiene una sensibilidad de $90,5 \%$ y especificidad de $83,3 \%$ con punto de corte de 24 . Conclusiones. La versión peruana adaptada del "eurotest", denominada "prueba de la moneda peruana" podría ser útil en el tamizaje de trastorno cognitivo de adultos mayores.
\end{abstract}

Palabras clave: Demencia; Ancianos; Tamizaje; Sensibilidad y especificidad (fuente: DECS BIREME).

\section{EVALUATION OF PERUVIAN MONEY TEST IN SCREENING OF COGNITIVE IMPAIRMENT AMONG OLDER ADULTS}

\begin{abstract}
Objectives. To evaluate the Peruvian adaptation of the money test (Eurotest) for identifying cognitive impairment among $>60$-year-old adults. Materials and methods. This is a phase I study of diagnostic test, with a convenience sampling and calculation of the test's sensitivity and specificity, based on a pretest prevalence of $50 \%$. The criteria of the Diagnostic and Statistical Manual of Mental Disorders (DSM IV) and Global Deterioration Scale (GDS) were used for the operational definition of patients with cognitive impairment. Receiver operating characteristic (ROC) curve analysis was used to identify the optimal cut-off value. Results. The study evaluated 42 cases and 42 controls; there was no significant difference between age (77.88 \pm 6.01 years vs. $6.4976 .14 \pm$ years) and years of education ( $13.69 \pm 3.70$ years vs. 8.17 \pm 4.71 years). The Peruvian version of the Eurotest has a sensitivity of $90.5 \%$ and specificity of $83.3 \%$ with cut-off value of 24. Conclusions. The Peruvian adapted version of the Eurotest, called prueba de la moneda peruana could be useful in screening for cognitive impairment among older adults.
\end{abstract}

Key words: Dementia; Aged; Screening; Sensitivity and specificity (source: MeSH NLM).

\section{INTRODUCCIÓN}

La demencia es un grave problema de salud pública en el mundo. La prevalencia mundial de demencia en personas mayores de 60 años es de $5-7 \%$; para América latina es $8,5 \%{ }^{(1)}$ y para el Perú es $3,1 \%(4,4 \%$ en mayores de 80 años) (2). El diagnóstico precoz es muy importante y, para ello, se requiere de instrumentos adecuados que detecten el trastorno cognitivo.

En la valoración cognitiva estructurada para la detección de síndrome demencial en las personas adultas mayores existen diversos instrumentos: pruebas acortadas o de detección (ejemplo: test de Pfeiffer, Short orientation-memory-concentration, Test of cognitive impairment, etc) (3); escalas de evaluación (Minimental Status Examination o MMSE) (4); escalas de deterioro (Dementia Rating Scale DRS, Geriatric Rating Scale, etc) ${ }^{(5)}$; escalas de gravedad de demencia (Global Deterioration Scale, Alzheimer Disease Assesment Scale, etc. ${ }^{(6)}$ y baterías de evaluación neuropsicológica (test de Barcelona, CERAD, CAMDEX, etc) ${ }^{(7,8)}$. Durante la evaluación integral del adulto mayor (personas mayores de 60 años) se emplean las pruebas acortadas

\footnotetext{
Centro de Investigación del Envejecimiento (CIEN), Instituto de Investigación, Facultad de Medicina, Universidad de San Martin de Porres. Lima, Perú.

Instituto de Investigación, Facultad de Medicina, Universidad de San Martin de Porres. Lima, Perú

Doctor en Medicina

Recibido: 03-08-15 Aprobado: 04-11-15
}

Citar como: Oscanoa TJ, Cieza E, Parodi JF, Paredes N. Evaluación de la prueba de la moneda peruana en el tamizaje de trastorno cognitivo en adultos mayores. Rev Peru Med Exp Salud Publica. 2016;33(1):67-73. doi: 10.17843/rpmesp.2016.331.2009 
o de detección y algunas escalas de evaluación como el Minimental state examination (MMSE) o test de Folstein (4), dado que su tiempo de aplicación es breve sirven para discriminar a aquellos pacientes que requieren de una evaluación cognoscitiva ulterior y en forma más detallada, con los otros exámenes neuropsicológicos disponibles.

En el Perú, el test de Pfeiffer (3) forma parte de una adenda la historia clínica, para la valoración geriátrica integral propuesta por Varela et al. ${ }^{\left({ }^{9}\right)}$. EI MMSE es el instrumento más usado en el tamizaje de demencia en adultos mayores y se ha constituido en un instrumento válido tanto en estudios clínicos como epidemiológicos.

De manera general, las pruebas mencionadas están diseñados para personas alfabetas, su utilidad es discutible en personas analfabetas o con muy bajo nivel educativo, existen muy pocas pruebas validadas en estas personas, uno de ellos es el "test del dinero" (10) creado por Carnero et al. en España, que inicialmente utilizada pesetas (las monedas en vigor en España) y posteriormente se adaptó al sistema monetario euro, denominándose en adelante "eurotest".

El eurotest (11) es una prueba breve, que evalúa la capacidad manipulativa y de cálculo con monedas de curso legal y diario. Existen estudios de su validación con monedas propias de algunos países. Iturra et al. ${ }^{(12)}$ validaron el eurotest en moneda chilena, encontrando una sensibilidad de $93 \%$ y una especificidad de $90 \%$, con una prevalencia pretest del $50 \%$. Además, existe el renminbi test que es la adaptación del eurotest a la moneda china ${ }^{(13)}$.

El presente estudio tiene el objetivo la adaptación del eurotest al contexto peruano (prueba de la moneda peruana), en el tamizaje del trastorno cognitivo en adultos mayores.

\section{MATERIALES Y MÉTODOS}

\section{DISEÑO DEL ESTUDIO}

El presente es un estudio de fase I de evaluación de prueba diagnóstica, con muestreo por conveniencia y cálculo de sensibilidad y especificidad, con una prevalencia pretest del $50 \%$. El estudio se realizó en el Hospital del Día de servicio de Geriatría del Hospital Guillermo Almenara Irigoyen, durante el periodo de agosto de 2012 a agosto de 2014 .

\section{POBLACIÓN}

Los pacientes captados para el estudio fueron aquellos que acudieron al hospital para una evaluación geriátrica integral que incluye valoración cognitiva. Los criterios de inclusión fueron: pacientes igual o mayor de 60 años que acudieron al servicio de Geriatría del hospital en el periodo de estudio y que aceptaron ingresar al estudio. Los criterios de exclusión fueron pacientes con trastorno sensorial (trastornos en la visión) y/o auditivo y que no aceptaron ingresar al estudio. Para la definición operacional de pacientes con trastorno cognitivo se utilizó los criterios de The Diagnostic and Statistical Manual of Mental Disorders (DSM IV) ${ }^{(14)}$ y el Global Deterioration Scale (GDS) ${ }^{(6)}$,

\section{DEFINICIÓN DE CASOS Y CONTROLES}

Los pacientes estudiados fueron 42 casos y 42 controles. Se denominó casos a aquellos pacientes que tenían trastorno cognitivo de acuerdo con los criterios de DSM IV y cuando el puntaje de GDS eran mayor de 2; se denominó controles a aquellos que no presentaban trastorno cognitivo según la DSM IV y tenían un GDS de 1 o 2. Los casos cumplieron los criterios del DSM IV, teniendo como evidencia una evaluación neuropsicológica previa en su historia clínica, con diagnóstico de trastorno cognitivo. De estos, 25 tenían diagnóstico por el test de Barcelona ${ }^{(7)}$ y en 17 pacientes se realizó el diagnóstico durante el estudio, utilizando el MMSE (versión en español de Lobo et al.) ${ }^{(15,16)}$. La media del GDS en los casos fue de 4,2 \pm 0,79 (rango: 3-6). En los controles la evaluación cognitiva se realizó con el MMSE, la media del puntaje fue de $27,57 \pm 1,50$ y la media del GDS fue de 1,71 $\pm 0,47$ (rango 1-2).

\section{PROCEDIMIENTO DE LA PRUEBA DE LA MONEDA PERUANA}

Para determinar la validez de contenido del instrumento, el proceso de adaptación de eurotest (11) a la prueba de la moneda peruana tuvo dos fases. La primera fase consistió en la adaptación de los reactivos (monedas en euros) en moneda peruana (3 de dos soles, 1 de un sol, 2 de 50 céntimos, 5 de 20 céntimos). La segunda fase consistió en la adaptación semántica de las preguntas del test, el estudio piloto determinó que era necesario cambiar una de las preguntas originales del test: ¿Me puede cambiar esta moneda (€ 1,00) por otras más pequeñas?, por la expresión más común en Perú: ¿Puede cambiarme en sencillo esta moneda $(S / 1,00)$ ?

La prueba de la moneda peruana al igual que el eurotest, consta de 3 partes (Anexo 1):

Parte I. Denominación de las monedas y billetes en curso: Ítem 1: denominación de monedas (0-6 puntos). Ítem 2: denominación de billetes (0-5 puntos). Forma de puntuar: un punto por respuesta correcta; restar un punto por cada intrusión (error) y mantener 0 puntos como mínimo en cada ítem. 
Puntuación de la parte I: 0-11 puntos. Tiempo máximo en los dos ítems: 1 minuto.

Parte II. Tareas de cálculo de complejidad creciente con las siguientes 11 monedas ( 3 de dos soles, 1 de un sol, 2 de 50 céntimos, 5 de 20 céntimos)

Ítem 1: contar las monedas (0-2 puntos).

Ítem 2: cambiar la moneda de 2 soles en moneda fraccionaria (0-2 puntos).

Ítem 3: sumar todas las monedas (0-2 puntos).

Ítem 4: dividir las monedas en dos montones que tengan el mismo dinero cada uno (0-2 puntos).

Ítem 5: dividir las monedas en tres montones que tengan el mismo dinero cada uno (0-2 puntos).

Forma de puntuar: 2 puntos si la respuesta inicial es correcta; en caso de respuesta errónea, hacerlo saber y dar una nueva oportunidad; en caso de respuesta correcta tras error, 1 punto; en caso de dos respuestas erróneas, 0 puntos.

Tiempo máximo de 1 minuto por ítem; si se supera este tiempo sin contestación, 0 puntos.

Puntuación de la parte II: 0-10 puntos.

Tarea de distracción. Tarea de fluidez verbal: animales en un minuto, siguiendo las instrucciones.

Parte III. Recuerdo de las monedas que se han manipulado previamente:

Ítem 1: recordar el número de monedas (0-1 puntos).

Ítem 2: recordar el dinero total (0-1 puntos).

Ítem 3: recordar el tipo y número de las monedas que ha manipulado (0-8 puntos)

Forma de puntuar: los ítems 1 y 2 se puntúan con 1 punto si se responden correctamente, y 0 puntos en caso contrario. En el ítem 3, dar 2 puntos si recuerda el tipo y la cantidad exactamente, y solo 1 punto en caso de que recuerde solo el tipo y no la cantidad o esta sea incorrecta. Restar 1 punto en este ítem en caso de intrusiones de tipo de monedas, hasta el mínimo de 0 puntos.
Puntuación de la parte III: 0-10 puntos.

Puntuación total de la prueba de la moneda peruana (suma total de la parte I, II y III): 0-31 puntos. Entre la parte II y III de la prueba hay una prueba de distracción (prueba de fluidez verbal) cuyo puntaje no se toma en cuenta.

\section{ANÁLISIS ESTADÍSTICO}

Todos los análisis fueron realizados con el paquete informático EPI INFO 2012 versión 3.5.4. Para el análisis estadístico comparativo de las variables consideradas, entre los grupos estudiados (casos y controles) se utilizó el test $t$ de Student para muestras independientes y apareadas o ANOVA de un factor para las variables cuantitativas y la prueba chi cuadrado para las categóricas. Se han estudiado distintos puntos de corte para encontrar el más idóneo, calculando para cada uno de ellos la sensibilidad (proporción de test positivos en pacientes con demencia), y especificidad (proporción de test negativos en sujetos sin la demencia). Adicionalmente, se determinó el punto de corte para la prueba de la moneda peruana mediante ploteo de los datos sobre la curva ROC (Receiver Operating Characteristic).

\section{CONSIDERACIONES ÉTICAS}

El presente estudio fue aprobado por el Comité de Ética en Investigación del Hospital Nacional Guillermo Almenara Irigoyen.

\section{RESULTADOS}

Los 84 pacientes adultos mayores (edad $\geq 60$ años) que ingresaron al estudio tuvieron las siguientes características demográficas: la media de edad de fue de $77,01 \pm 6,28$, con un rango entre 60 y 90 años; el sexo femenino estuvo presente en 54 (64,3\%), mientras que el masculino fue $30(35,7 \%)$, los años de educación fueron en promedio de $6,98 \pm 4,38$ (rango de 0 a 17 años) (Tabla 1).

Tabla 1. Prueba de la moneda peruana: características demográficas y resultados de exámenes de evaluación cognitiva

\begin{tabular}{lccc}
\hline \multicolumn{1}{c}{ Característica } & Casos $(\mathbf{n}: \mathbf{4 2})$ & Controles (n:42) & Valor $\mathbf{p}$ \\
\hline Edad (media y rango) & $\begin{array}{l}77,88+6,01 \\
\text { (Rango: } 60-90 \text { años) }\end{array}$ & $\begin{array}{c}76,14+6.49 \\
\text { (Rango: } 60-90 \text { años) }\end{array}$ & No significativo \\
Sexo & $\begin{array}{l}\text { Femenino: } 24(57,1 \%) \\
\text { Masculino: } 18(42,9 \%)\end{array}$ & $\begin{array}{c}\text { Femenino: } 30(71,4 \%) \\
\text { Masculino: } 12(28,6 \%)\end{array}$ & No significativo \\
Grado de instrucción & $13,69 \pm 3,70$ & $8,17 \pm 4,71$ & No significativo \\
MMSE* & Rango:0-17 años & Rango: $2-17$ años & p $<0,001$ \\
Prueba de la moneda peruana & $17,76 \pm 5.78$ & $27,57 \pm 1.50$ & p $<0,001$ \\
\hline $\begin{array}{l}\text { Tiempo de aplicación de prueba } \\
\text { de la moneda peruana }\end{array}$ & $16,74 \pm 5,96$ & $26,38 \pm 3,19$ & No significativo \\
\hline
\end{tabular}

* Mini Mental State Examination, versión en español de Lobo et al. ${ }^{(15,16)}$. Puntaje máximo 30, deterioro cognitivo < 24 
El puntaje promedio del MMSE en los casos fue de 17,76 $\pm 5,78$ y en los controles fue de $27,57 \pm 1,50(p<0,001)$. Al comparar los resultados de la prueba de la moneda peruana con el MMSE, se observó una buena correlación (coeficiente de correlación de Pearson: 0,73). El puntaje de la prueba de la moneda peruana en los casos fue de $16,74 \pm 5,96$ y en los controles fue de $26,38 \pm 3,19$ $(p<0,001)$. Mediante la aplicación de diferentes puntos de corte en una curva ROC (Receiver Operating Characteristic) se determinó que el mejor punto de corte fue 24, punto en el cual la sensibilidad fue de $90,5 \%$ y especificidad de $83,3 \%$ (Tabla 2 ).

\section{DISCUSIÓN}

En el presente estudio, la prueba de la moneda peruana (adaptación peruana del eurotest) presentó una alta sensibilidad y una alta especificidad.

La prueba de la moneda peruana, presenta características que pueden convertirlo en una buena alternativa (sería la única opción, teniendo en cuenta que no hay otros instrumento validados en el país) para el tamizaje de trastornos cognitivos en adultos mayores con bajo nivel educativo o analfabetos. Es fácil de aplicar, tiene instrucciones muy concretas y precisas, que minimizan las interpretaciones por parte del médico que lo aplica. Requiere escaso material (monedas y un formato autoexplicativo) y un tiempo máximo de aplicación de nueve minutos.

La población analfabeta es reacia a pruebas que incluyen lápiz y papel, la prueba de la moneda peruana tiene validez ecológica, es decir, que los pacientes son sometidos a pruebas de manipulación diaria y rutinaria de monedas y billetes actuales. La familiaridad con el sistema monetario es independiente del nivel educativo y del grado de alfabetización, esto es una ventaja importante en relación con otras pruebas de tamizaje

Tabla 2. Prueba de la moneda peruana: sensibilidad y especificidad para distintos puntos de corte

\begin{tabular}{ccc}
\hline $\begin{array}{c}\text { Punto de } \\
\text { corte }\end{array}$ & Sensibilidad (\%) & Especificidad (\%) \\
\hline$\geq 26$ & 1 & 0,59 \\
$\geq 25$ & 0,95 & 0,67 \\
$\geq 24^{*}$ & 0,90 & 0,83 \\
$\geq 23$ & 0,81 & 0,88 \\
$\geq 22$ & 0,71 & 0,93 \\
$\geq 21$ & 0,62 & 0,98 \\
$\geq 20$ & 0,59 & 0,98 \\
$\geq 19$ & 0,57 & 1 \\
\hline
\end{tabular}

* Mejor punto de corte

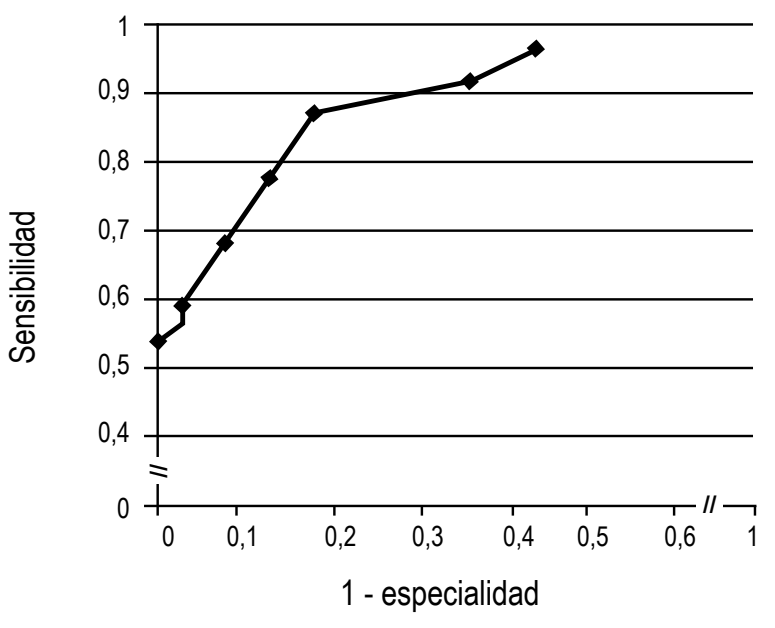

Figura 1. Curva ROC (Receiver Operating Characteristic) de la prueba de la moneda peruana

como el MMSE (4), SPMSQ (short portable mental status questionnaire) ${ }^{(3)}$, test del reloj ${ }^{(17,18)}$ y el test de los siete minutos (T7M) ${ }^{(19)}$. En un estudio multicéntrico, el eurotest mostró una sensibilidad del $91 \%$ y una especificidad del $82 \%{ }^{(20)}$.

El presente estudio presenta algunas limitaciones, propias de un estudio de test diagnóstico de fase I, los cuales están destinados básicamente a evaluar la factibilidad de un test diagnóstico en un contexto determinado; en este tipo de estudios el objetivo principal es el proceso de adaptación transcontextual del test, es decir, determinar la validez de contenido, entre las cuales están la adaptación de los reactivos (adaptación de la moneda original del test, euros a la moneda peruana) y la adaptación semántica de las preguntas del instrumento al contexto nacional. Se hace necesario estudios más extensos, con validación prospectiva (diseño de cohorte), estudios de impacto (estudio de ensayo clínico controlado y, finalmente, con evaluación de fiabilidad (test-retest, evaluadores múltiples). Adicionalmente, hacen falta estudios comparativos con otros test de tamizaje destinado a analfabetos, entre estos últimos exámenes están el foto-test o test de la fotografía ${ }^{(21)}$ y el Memory Alteration Test ${ }^{(22)}$. De igual manera, es necesario evaluar la influencia del idioma (investigaciones en quechua hablantes y otros dialectos del país).

En conclusión, la presente investigación cuyo objetivo fue la adaptación del eurotest al contexto peruano, ha mostrado que la prueba de la moneda peruana tiene una aceptable sensibilidad y especificidad en el tamizaje de trastorno cognitivo en adultos mayores.

Agradecimientos: al Dr. Cristóbal Carnero Pardo del Servicio de Neurología del Hospital Universitario Virgen de las Nieves 
en Granada, España. A la Dra. Luz Pecho Sánchez, jefa del Servicio de Geriatría del Hospital Guillermo Almenara Irigoyen y a todos los médicos asistentes, residentes y profesionales de la salud, por su invalorable colaboración en el proyecto.

Contribuciones de autoría: TJO participo en la concepción y diseño del artículo, análisis e interpretación de datos, redacción del artículo y aprobación de su versión final. EC participó en la recolección / obtención de resultados y aporte de pacientes o material de estudio. JP participó en la revisión crítica del artículo y obtención de financiamiento. NP participó en la revisión crítica del artículo, asesoría estadística y asesoría técnica o administrativa.

Fuentes de financiamiento: Centro de Investigación del Envejecimiento (CIEN) del Instituto de Investigación de la Facultad de Medicina de la Universidad de San Martin de Porres. Lima, Perú

Conflictos de interés: los autores declararan no tener conflictos de interés, que puedan reducir la objetividad en la interpretación del presente trabajo de investigación.

\section{REFERENCIAS BIBLIOGRÁFICAS}

1. Prince M, Bryce R, Albanese E, Wimo A, Ribeiro W, Ferri CP.The global prevalence of dementia: a systematic review and metaanalysis. Alzheimers Dement. 2013;9(1):63-75.e2. doi: 10.1016/j.jalz.2012.11.007.

2. Sosa AL, Albanese E, Stephan BC, Dewey M, Acosta D, Ferri CP, et al. Prevalence, distribution, and impact of mild cognitive impairment in Latin America, China, and India: a 10/66 population-based study. PLoS Med. 2012;9(2):e1001170. doi: 10.1371/ journal.pmed.1001170.

3. Pfeiffer E.A short portable mental status questionnaire for the assessment of organic brain deficit in elderly patients. J Am Geriatr Soc. 1975;23(10):433-41.

4. Folstein MF, Folstein SE, McHugh PR."Mini-mental state". A practical method for grading the cognitive state of patients for the clinician. J Psychiatr Res. 1975;12(3):189-9

5. Hughes CP, Berg L, Danziger WL, Coben LA, Martin RL. A new clinical scale for the staging of dementia. $\mathrm{Br} \mathrm{J}$ Psychiatry. 1982;140:566-72.

6. Reisberg B, Ferris SH, de Leon MJ, Crook T. The Global Deterioration Scale for assessment of primary degenerative dementia. Am J Psychiatry. 1982;139(9):1136-9.

7. Peña-Casanova J, Guardia J, BeltránSierra E, Manero RM, Espel G, Martin A, et al. "Versión abreviada del test Barcelona (III): validez de criterio con el ADAS-Cog". Neurologia.1997;12(3):117-9.

8. Welsh KA, Butters N, Mohs RC, Beekly D, Edland S, Fillenbaum G, et al. The Consortium to Establish a Registry for Alzheimer's Disease (CERAD). Part V. A normative study of the neuropsychological battery.
Neurology. 1994;44(4):609-14.

9. Varela LF, Silicani A, Chavez H, Chigne O. Valoración Geriátrica Integral: propuesta de addendum a la Historia clínica. Diagnostico (Peru). 2000;39(3):135-47.

10. Carnero C, Barquero MS, Payno MA, Frank A, Baquero M, Lacruz F, et al. The Money Test. Neurology. 2002;58:A274.

11. Carnero-Pardo C, Montoro-Ríos MT. [Preliminary evaluation of a new screening test for dementia (Eurotest)]. Rev Neurol. 2004;38(3):201-9. [Spanish]

12. Iturra-Mena AM. Adaptación y validación preliminar de un test para el screening de demencia en Chile: El Eurotest. Rev Chil Neuro-Psiquiat. 2007;45:296-304.

13. Ding D, Zhao Q, Guo Q, Meng H, Wang B, Luo J, et al. Prevalence of mild cognitive impairment in an urban community in China: a cross-sectional analysis of the Shanghai Aging Study. Alzheimers Dement. 2015;11(3):3009.e2. doi: 10.1016/j.jalz.2013.11.002.

14. DSM-IV. Manual diagnóstico y estadístico de los trastornos mentales. Barcelona: Masson; 1995.

15. Lobo A, Saz P, Marcos G, Día J L, de la Cámara C, Ventura $\mathrm{T}$, et al. Revalidación y normalización del mini-examen cognoscitivo (primera versión en castellano del Mini-Mental Status Examination) en la población general geriátrica. Med Clin (Barc) 1999;112(20):767-74.

16. Lobo A, Saz P, Marcos G, Grupo de Trabajo ZARADEMP. MMSE: Examen cognoscitivo Mini-Mental. Madrid: TEA Ediciones; 2002.

17. Agrell B, Dehlin O. The clock-drawing test. 1998. Age Ageing. 2012;41 Suppl 3:iii41-5. doi: 10.1093/ageing/afs149.
18. Oscanoa T. Evaluación de la prueba del reloj en el tamizaje de enfermedad de Alzheimer. An Fac Med (Lima). 2004;65(1):42-8.

19. Solomon PR, Hirschoff A, Kelly B, Relin M, Brush M, DeVeaux RD, et al. A 7 minute neurocognitive screening battery highly sensitive to Alzheimer's disease. Arch Neurol. 1998;55(3):34955.

20. Carnero-Pardo C, Gurpegui M, Sanchez-Cantalejo E, Frank A, Mola $\mathrm{S}$, Barquero MS, et al. Diagnostic accuracy of the Eurotest for dementia: a naturalistic, multicenter phase II study. BMC Neurol. 2006;6:15. doi: 10.1186/1471-377-6-15.

21. Carnero-Pardo C, Montoro-Ríos MT. [The photo test]. Rev Neurol. 2004;39(9):801-6

22. Rami L, Molinuevo JL, Sanchez-Valle R, Bosch B, Villar A. Screening for amnestic mild cognitive impairment and early Alzheimer's disease with M@T (Memory Alteration Test)in the primary care population. Int J Geriatr Psychiatry. 2007;22(4):294-304.

Correspondencia: Teodoro Julio Oscanoa Espinoza

Dirección: Hospital Almenara. Av. Grau 800. Lima 13. Perú.

Teléfono: (511)324 2983 anexo 44081

Correo electrónico: tjoscanoae@gmail.com;

toscanoae@usmp.pe 


\section{ANEXO 1}

Adaptación del eurotest: test de la moneda peruana

\section{Primera parte (Ítem 1 y 2: conocimiento/denominación)}

“¿Recuerda de qué cantidades hay monedas en la actualidad?; fijese que le pregunto monedas y no billetes” (máximo un minuto)

Céntimos 10( ) 20 ( ) 50 ( ) Soles 1 ( ) 2 soles( ) 5 soles ( )

Otras (especificar): total correctas: Intrusiones:

"¿Recuerda de qué cantidades hay billetes actualmente?" (máximo un minuto)

Soles: $10($ ) 20 () 50 () 100 () 200 ()

Otras (especificar): total correctas: Intrusiones:

\section{Segunda parte (Ítems 3 a 7: calculo con monedas)}

Ponga delante del sujeto las monedas ( 3 de dos soles, 1 de un sol, 2 de 50 céntimos, 5 de 20 céntimos) y pídale de forma consecutiva las siguientes tareas. En caso de que la contestación sea errónea, hágaselo saber y dele un único nuevo intento. Algunos ítems tienen varias soluciones, todas ellas son válidas. Conceda un máximo de un minuto por cada ítem y pase al siguiente si no ha contestado correctamente en este tiempo o ha agotado los dos intentos.

¿Cuántas monedas hay aquí? (11)

Correcto ( ) Correcto segundo intento ( ) incorrecto ( )

"¿Puede cambiarme en sencillo esta moneda?"

(retirar las 2 moneda de 2 soles y mostrarla solo una)

Correcto ( ) Correcto segundo intento ( ) incorrecto ( )

“¿Cuánto dinero hay aquí en total?" (9 soles) (de nuevo todas las monedas)

Correcto ( ) Correcto segundo intento ( ) incorrecto ( )

Por favor, reparta estas monedas en dos montones que tengan el mismo dinero (4,50 soles)

Correcto ( ) Correcto segundo intento ( ) incorrecto ( )

Ídem, en tres montones que tengan el mismo dinero (3 soles)

Correcto ( ) Correcto segundo intento ( ) incorrecto ( )

3. Tarea de distracción: fluencia verbal semántica (60 segundos)

"Quiero que me diga todos los nombres de animales que se le ocurran, ya sean de la tierra, del mar o del aire, del campo o de la casa, ijtodos los que se le ocurran!!"

4.- Tercera parte (recuerdo)

"Para finalizar, quiero que haga un último esfuerzo y trate de recordar":

\begin{tabular}{|c|c|c|c|c|}
\hline \multicolumn{4}{|c|}{$\begin{array}{l}\text { “¿Cuántas monedas le enseñé antes?” (11) } \\
\text { Respuesta: correcto ( ) incorrecto () }\end{array}$} & \multirow{8}{*}{ C } \\
\hline \multicolumn{4}{|c|}{$\begin{array}{l}\text { “¿Cuánto dinero había en total?” (9 soles) } \\
\text { Respuesta: correcto ( ) incorrecto ( ) }\end{array}$} & \\
\hline \multicolumn{4}{|c|}{ “¿Recuerda qué monedas había exactamente?” } & \\
\hline Cantidad & Moneda & Intrusiones & Total intrusiones & \\
\hline $5 \mathrm{de}$ & 20 céntimos & & & \\
\hline $2 \mathrm{de}$ & 50 céntimos & & & \\
\hline $1 \mathrm{de}$ & $1 \mathrm{sol}$ & & & \\
\hline $3 \mathrm{de}$ & 2 soles & & & \\
\hline
\end{tabular}

\section{Observaciones:}




\section{ANEXO 2}

La escala global del deterioro para la evaluación de la demencia primaria degenerativa (GDS) (también conocida como la escala de Reisberg)

\begin{tabular}{|c|c|c|}
\hline Diagnóstico & Fase & Señales y síntomas \\
\hline $\begin{array}{l}\text { Falta de } \\
\text { demencia }\end{array}$ & $\begin{array}{c}\text { Fase 1: } \\
\text { Ningún declive cognitivo }\end{array}$ & $\begin{array}{l}\text { En esta fase la persona tiene una función normal, no experimenta la pérdida } \\
\text { de la memoria, y es sano mentalmente. Gente que no tiene la demencia sería } \\
\text { considerada en la Fase } 1 .\end{array}$ \\
\hline $\begin{array}{l}\text { Falta de } \\
\text { demencia }\end{array}$ & $\begin{array}{l}\text { Fase 2: } \\
\text { Un declive cognitivo muy } \\
\text { leve }\end{array}$ & $\begin{array}{l}\text { Esta fase se usa para describir el olvido normal asociado con el envejecimiento; } \\
\text { por ejemplo, olvidarse de los nombres y de donde se ubican los objetos familiares. } \\
\text { Los síntomas no son evidentes a los seres queridos ni al médico. }\end{array}$ \\
\hline $\begin{array}{l}\text { Falta de } \\
\text { demencia }\end{array}$ & $\begin{array}{c}\text { Fase 3: } \\
\text { Declive cognitivo leve }\end{array}$ & $\begin{array}{l}\text { Esta etapa incluye la falta de memoria creciente, dificultad leve que concentra, } \\
\text { funcionamiento de trabajo disminuido. La gente puede conseguir perdió más a } \\
\text { menudo o tiene dificultad que encuentra las palabras correctas. En esta etapa, } \\
\text { las personas amadas comenzarán a notar una declinación cognoscitiva. Duración } \\
\text { media: } 7 \text { años antes del inicio de la demencia }\end{array}$ \\
\hline $\begin{array}{l}\text { Etapa } \\
\text { temprana }\end{array}$ & $\begin{array}{l}\text { Fase 4: } \\
\text { Declive cognitivo } \\
\text { moderado }\end{array}$ & $\begin{array}{l}\text { Esta etapa incluye dificultades de concentrarse, una disminución de la habilidad } \\
\text { de acordarse de los eventos recientes, y dificultades de manejar las finanzas } \\
\text { o de viajar solo a lugares nuevos. La gente tiene problemas llevando a cabo } \\
\text { eficientemente/con precisión las tareas complejas. Puede no querer reconocer } \\
\text { sus síntomas. También la gente puede recluirse de los amigos y de la familia } \\
\text { porque las interacciones sociales se hacen más difíciles. En esta etapa un médico } \\
\text { puede notar problemas cognitivos muy claros durante una evaluación y entrevista } \\
\text { con el paciente. Duración promedio: } 2 \text { años. }\end{array}$ \\
\hline Etapa media & $\begin{array}{l}\text { Fase 5: } \\
\text { Declive cognitivo } \\
\text { moderadamente severo }\end{array}$ & $\begin{array}{l}\text { Gente en esta fase tiene deficiencias serias de la memoria y necesita ayuda } \\
\text { a completar las actividades diarias (vestirse, bañarse, preparar la comida). } \\
\text { La pérdida de la memoria se destaca más que antes y puede incluir aspectos } \\
\text { importantes de la vida actual; por ejemplo, puede ser que la persona no recuerda } \\
\text { su domicilio o número de teléfono. También puede que no sepa la hora, el día, o } \\
\text { donde está. Duración promedio: } 1,5 \text { años. }\end{array}$ \\
\hline Etapa media & $\begin{array}{l}\text { Fase 6: } \\
\text { Declive cognitivo severo } \\
\text { (la demencia media) }\end{array}$ & $\begin{array}{l}\text { Las personas en esta fase requieren ayuda extensiva a hacer las actividades } \\
\text { diarias. Empiezan a olvidar los nombres de los miembros de la familia y tienen } \\
\text { muy poco recuerdo de los eventos recientes. Muchas personas solamente } \\
\text { pueden recordar algunos detalles de la vida temprana. También tienen dificultades } \\
\text { de contar atrás de } 10 \text { y de llevar a cabo las tareas. La incontinencia (la pérdida del } \\
\text { control de la vejiga o de los intestinos) es un problema en esta fase. Cambios de la } \\
\text { personalidad tales como el delirio (creer algo que no es verdad), las compulsiones } \\
\text { (repetir una actividad, como limpiar), la ansiedad o la agitación pueden ocurrir. } \\
\text { Duración promedio: } 2,5 \text { años. }\end{array}$ \\
\hline $\begin{array}{l}\text { Etapa } \\
\text { avanzada }\end{array}$ & $\begin{array}{l}\text { Fase } 7: \\
\text { Declive cognitivo muy } \\
\text { severo (la demencia } \\
\text { avanzada) }\end{array}$ & $\begin{array}{l}\text { Las personas en esta fase esencialmente no tienen la habilidad de hablar ni de } \\
\text { comunicarse. Requieren ayuda con la mayoría de las actividades (p.ej., usar el } \\
\text { baño, comer). A menudo pierden las habilidades psicomotores, por ejemplo la } \\
\text { habilidad de caminar. Duración promedio: } 2,5 \text { años. }\end{array}$ \\
\hline
\end{tabular}

De: Reisberg, Barry, et al. "The Global Deterioration Scale for assessment of primary degenerative dementia". The American Journal of Psychiatry (1982). 